Metzler Lexikon Literatur- und Kulturtheorie 


\section{Metzler Lexikon \\ Literatur- und Kulturtheorie}

Ansätze - Personen - Grundbegriffe

Herausgegeben von Ansgar Nünning

2., überarbeitete und erweiterte Auflage

Verlag J. B. Metzler

Stuttgart - Weimar 
Inhaltsverzeichnis

$\begin{array}{ll}\text { Vorwort } & \text { S. V-VII } \\ \text { Vorwort zur zweiten Auflage } & \text { S. VIII-IX } \\ \begin{array}{l}\text { Artikel A-Z } \\ \text { Allgemeine Abkürzungen }\end{array} & \text { S. 1-691 } \\ \begin{array}{l}\text { Verzeichnis der Mitarbeiterinnen } \\ \quad \text { und Mitarbeiter }\end{array} & \\ \begin{array}{l}\text { Auswahlbibliographie literatur- } \\ \quad \text { und kulturtheoretischer Werke }\end{array} & \text { S. } 694 \\ & \text { S. } 696\end{array}$

Die Deutsche Bibliothek - CIP-Einheitsaufnahme

Metzler-Lexikon Literatur- und Kulturtheorie : Ansätze - Personen - Grundbegriffe /

hrsg. von Ansgar Nünning. - 2., überarb. und erw. Aufl. - Stuttgart ; Weimar : Metzler, 2001

ISBN 978-3-476-01692-8

ISBN 978-3-476-01692-8

ISBN 978-3-476-03688-9 (eBook)

DOI 10.1007/978-3-476-03688-9

Dieses Werk einschließlich aller seiner Teile ist urheberrechtlich geschützt. Jede Verwertung außerhalb der engen Grenzen des Urheberrechtsgesetzes ist ohne Zustimmung des Verlages unzulässig und strafbar. Das gilt insbesondere für Vervielfältigungen, Übersetzungen, Mikroverfilmungen und die Einspeicherung und Verarbeitung in elektronischen Systemen.

(C) 2001 Springer-Verlag GmbH Deutschland

Ursprünglich erschienen bei J. B. Metzlersche Verlagsbuchhandlung

und Carl Ernst Poeschel Verlag GmbH in Stuttgart 2001

www.metzlerverlag.de

info@ metzlerverlag.de 


\section{Vorwort}

Kein Teilbereich der Literatur- und Kulturwissenschaften hat in den letzten Jahrzehnten einen ähnlichen Boom erlebt wie die Theoriebildung, die die Entwicklung aller Philologien betrifft. Die seit Ende der 1960er Jahre erhobene Forderung nach einer stärkeren Theoretisierung der Geisteswissenschaften hat inzwischen zur Entwicklung einer Vielzahl literatur- und kulturwissenschaftlicher Theorien, Modelle und Methoden geführt. Ob es sich dabei um eine begrüßens- oder beklagenswerte Entwicklung handelt, mag zwar eine Frage sein, über die sich trefflich streiten läßt, unstrittig ist jedoch zweierlei. Erstens hat sich inzwischen die Einsicht durchgesetzt, daß jede Form von Erkenntnis, Beobachtung und Interpretation theoriegeleitet ist. Der von Theoriegegnern gern herausgestellte Gegensatz zwischen einem theorielastigen und einem sdirekten s oder sunverstellten Zugang zu literarischen Texten erweist sich daher als eine falsch formulierte Alternative: Die Frage lautet nicht, $o b$ sich Literatur- und Kulturwissenschaftler bestimmter Theorien, Modelle und Konzepte bedienen oder nicht, sondern wie bewußt sie sich ihrer theoretischen und methodischen Prämissen sind und wie explizit sie die verwendeten Kategorien darlegen. Zweitens beweist ein Blick in die Vorlesungsverzeichnisse und Prüfungsordnungen der Universitäten, daß die Darstellung theoretischer Grundlagen der Literatur- und Kulturwissenschaften in Lehrveranstaltungen eine immer größere Rolle spielt und daß Studierende sämtlicher Philologien inzwischen gar nicht mehr umhin können, sich mit literatur- und kulturwissenschaftlichen Theorien, Begriffen und Methoden vertraut zu machen.

Das Lexikon Literatur- und Kulturtheorie ist als Arbeitsmittel für die Orientierung innerhalb eines Sach- und Begriffsfeldes konzipiert, das inzwischen selbst für Fachleute kaum noch überschaubar ist. Das interdisziplinär ausgerichtete Lexikon gibt einen kompakten Überblick über die Vielfalt der literatur- und kulturwissenschaftlichen Ansätze, erläutert die zentralen Grundbegriffe und verschafft einen Zugang zu den Autor/innen, die die theoretischen Debatten bestimmt haben. Es soll Studierenden (auch Studienanfänger/innen) aller Philologien und Kulturwissenschaften sowie Wissenschaftler/innen und theorieinteressierten Leserinnen und Lesern anderer geisteswissenschaftlicher Disziplinen (insbesondere Historikern, Soziologen und Psychologen) fachliche Orientierungshilfe bieten und ihnen ermöglichen, sich innerhalb des interdisziplinären Diskussionszusammenhangs der Literatur- und Kulturtheorie eine erste begriffliche Übersicht zu verschaffen. Um diesem Anspruch zu genügen, muß das Lexikon so umfassend wie möglich sein, ohne seine Handlichkeit als einbändiges Nachschlagewerk zu verlieren.

Das Lexikon Literatur- und Kulturtheorie kommt mit seiner Zusammenschau von theoretischen Ansätzen, Autor/innen und Grundbegriffen dem wachsenden Bedürfnis nach Orientierungswissen entgegen und macht, über die prägnante Information über einzelne Begriffe und Personen hinausgehend, Zusammenhänge transparent. Im Gegensatz zu reinen Autoren-, Begriffs- und Werklexika bietet dieses Lexikon in über 600 Artikeln verständliche und zuverlässige Einführungen in die wichtigsten literaturund kulturwissenschaftlichen Ansätze, deren Hauptrepräsentanten und die von ihnen geprägten Grundbegriffe. Im Mittelpunkt der Artikel stehen die Charakterisierung der theoretischen Grundlagen der verschiedenen Ansätze und die Erläuterung der jeweils relevanten Konzepte sowie der methodischen Zugangsmöglichkeiten zur Analyse von literarischen und kulturellen Phänomenen.

Im Zentrum dieses Lexikons steht die moderne Literatur- und Kulturtheorie, die durch literaturgeschichtliche Überblicksartikel (u.a. zu Literaturtheorien der Antike, des Mittelalters, der Renaissance, des Klassizismus, der Romantik, des Realismus, des Ästhetizismus sowie des Modernismus und Postmodernismus) und durch die Berücksichtigung wissenschaftsgeschichtlicher Aspekte in vielen anderen Beiträgen auch in ihrer historischen Entwicklung erschlossen wird. Neben textzentrierten und eher traditionellen Methoden wird eine Vielzahl von autoren-, leser- und kontextorientierten Ansätzen in einem internationalen und interdisziplinären Kontext vorgestellt. Umfassend berücksichtigt wurden vor allem auch neuere Entwicklungen wie Dekonstruktion, Diskurstheorie, feministische Theorien und Geschlechterforschung, Konstruktivismus, New Historicism, 
Mentalitätsgeschichte, postkoloniale Literaturkritik und Poststrukturalismus.

Der allseits geforderten - und zum Teil bereits vollzogenen - Weiterentwicklung der Philologien zu einer Kultur- und/oder Medienwissenschaft hin sowie der nicht zuletzt daraus resultierenden zunehmenden Interdisziplinarität der Theoriebildung wird dadurch Rechnung getragen, daß das Lexikon auch jene theoretischen Ansätze einbezieht, die in anderen geisteswissenschaftlichen Disziplinen entwickelt und später - oftmals eklektisch - in die Literaturwissenschaft übernommen wurden. Als Beispiele für solche übergreifenden Theorieentwürfe, die inzwischen auch die literatur- und kulturtheoretischen Diskussionen prägen, seien etwa Jacques Lacans Psychoanalyse, Michel Foucaults historische Diskursanalyse, Pierre Bourdieus Gesellschaftstheorie, Jacques Derridas Sprachkritik und Dekonstruktion sowie Niklas Luhmanns Systemtheorie genannt. Ebenso sind kultur- und medienwissenschaftlichen Ansätzen und Konzepten viele eigene Artikel gewidmet. Beiträge zu Themen wie "Film und Literatur", "Kunst und Literatur" "Musik und Literatur", "Photographie und Literatur", "Historiographie und Literatur" sowie "Naturwissenschaft und Literatur" tragen dazu bei, die zunehmend intermediale und interdisziplinäre Dimension der zeitgenössischen Literatur- und Kulturtheorie zu erschließen.

Darüber hinaus werden die wichtigsten Repräsentant/innen einer Vielzahl literatur- und kulturtheoretischer Ansätze sowie ihre Werke in Autorenporträts vorgestellt. Das breite Spektrum der Theoretiker/innen reicht von Aristoteles über Bachtin, de Man, Derrida, Foucault, Greenblatt, Iser, Jauß, Kristeva, Luhmann und Lyotard bis zu Virginia Woolf. Die Auswahl der Personen, die natürlich (ebenso wie jede andere) angreifbar ist, orientiert sich primär an der Bedeutung, die die Autorinnen bzw. Autoren für die zeitgenössischen Theoriedebatten haben; diese ist zwar schwer exakt abzuschätzen, aber die Register einschlägiger literatur- und kulturtheoretischer Publikationen neueren Datums oder der Humanities Citation Index bieten durchaus verläßliche Anhaltspunkte. Hinweise zur Rezeptions- und Wirkungsgeschichte der Werke der vorgestellten Theoretiker/innen sowie Querverweise zwischen den Artikeln erleichtern die wissenschaftsgeschichtliche Einordnung. Das Lexikon kann freilich nicht die Beschäftigung mit den Werken und dem Denken der Literatur- und Kulturtheoretiker/innen ersetzen, sondern will Leserinnen und Lesern einen verläßlichen Leitfaden für die bessere Orientierung geben.

Außerdem werden die zentralen Grundbegriffe der Literatur- und Kulturtheorie (von Appellfunktion bis Zirkulation) in über 300 kurzen Sachbegriffsartikeln definiert. Bei der Auswahl der Lemmata galt es, ein möglichst breites Spektrum abzudecken, die Überrepräsentierung von Konzepten, die mit bestimmten Ansätzen verbunden sind, zu vermeiden und vor allem jene Begriffe der Literatur- und Kulturtheorie zu erläutern, die in den etablierten Lexika der literarischen Terminologie (z.B. in G. Schweikle/I. Schweikle: Metzler Literatur Lexikon, Stuttgart: Metzler, 1984) weitgehend unberücksichtigt bleiben. Der Akzent liegt daher nicht auf jenen Begriffen aus den Bereichen der Poetik, Rhetorik, Metrik, Literaturgeschichte oder Gattungstheorie, deren Bedeutung in vielen Begriffslexika nachgeschlagen werden kann, sondern auf der Erläuterung zentraler Konzepte der modernen Literatur- und Kulturtheorie wie "Dialogizität", "Différance","Écritureféminine «,»Fokalisierung «, "Impliziter Leser" usw., ohne deren Kenntnis ein Großteil der Forschungsliteratur nicht mehr verständlich ist.

$\mathrm{Da}$ die literatur- und kulturwissenschaftliche Theoriebildung einen eminent dialogischen Prozeß darstellt, soll die Vielzahl interner Querverweise ( $\boldsymbol{\nearrow}$ ) zwischen den Sachbegriffs- und Autorenartikeln die Einordnung der Ansätze, Theoretiker/innen und Kategorien in übergeordnete systematische und wissenschaftsgeschichtliche Zusammenhänge erleichtern. Zugleich sind die einzelnen Begriffserläuterungen und Autorenporträts jedoch so abgefaßt, daß sie jeweils für sich selbst verständlich sein sollen, ohne daß der/die Leser/in durch die Verweise auf andere, verwandte Artikel zu ständigem Weiterblättern genötigt wäre.

Alle Artikel enthalten aktuelle Hinweise auf einführende oder weiterführende Literatur neueren Datums, die den Einstieg in das Werk des einzelnen Theoretikers bzw. den dem jeweiligen Ansatz oder Begriff zugehörigen Problembereich gezielt erleichtern sollen. Diese (notgedrungen selektiven) Auswahlbibliographien streben nicht nach Vollständigkeit, sondern sollen das Bedürfnis von Leserinnen und Lesern nach gezielter und kompakter Information und überblicksartiger Orientierung in einem für alle Philologien zentralen Bereich befriedigen. Für ausführlichere Bibliographien und umfassendere Darstellungen literatur- und kulturwissenschaft- 
licher Ansätze, Theoretiker/innen und Begriffe sei auf die in der Auswahlbibliographie (am Ende des Bandes) enthaltenen Titel verwiesen. Besonders hervorgehoben seien die folgenden vier Nachschlagewerke, die auch in vielen Artikeln des Lexikons zur Anwendung kamen: Encyclopedia of Contemporary Literary Theory: Approaches, Scholars, Terms, hrsg. von Irena R. Makaryk (Toronto: University of Toronto Press, 1993); The Johns Hopkins Guide to Literary Theory and Criticism, hrsg. von Michael Groden und Martin Kreiswirth (Baltimore, MD/ London: Johns Hopkins University Press, 1994); A Dictionary of Cultural and Critical Theory, hrsg. von Michael Payne (Oxford: Blackwell, 1996); Reallexikon der Deutschen Literaturwissenschaft, Bd. 1: A-G, hrsg. von Klaus Weimar (Berlin/New York, NY: de Gruyter, 1997). Alle in der Schlußbibliographie (mit vollständigen bibliographischen Angaben) genannten Publikationen zur Literatur- und Kulturtheorie sind - zur Entlastung des Gesamtumfangs des Bandes - in den Literaturangaben der einzelnen Artikel nur mit dem Namen der jeweiligen Autor/innen bzw. Herausgeber/innen und Jahreszahl aufgeführt.

Am Ende eines erquicklichen Gemeinschaftsunternehmens sei allen herzlich gedankt, die zu dessen Gelingen beigetragen haben. An erster Stelle möchte ich den Autorinnen und Autoren, die die Artikel für dieses Lexikon geschrieben haben, für die ertragreiche und zuverlässige $\mathrm{Zu}$ sammenarbeit danken. Ohne deren Disziplin und geduldige Bereitschaft, umgehend auf Rückfragen, Vorschläge und Kürzungswünsche zu reagieren, wäre das termingerechte Erscheinen dieses Lexikons nicht möglich gewesen. Darüber hinaus haben mir viele weitere Kolle- ginnen und Kollegen (zu viele, um sie namentlich zu nennen) in der Phase der Vorbereitung mit ihrer großen Belesenheit und mit gezielten Ratschlägen weitergeholfen. Sodann gilt der (kollektive) Dank Bernd Lutz und Ute Hechtfischer vom Metzler Verlag, die sich von Beginn an für dieses Projekt engagiert, es (bzw. den Herausgeber, seine Mitarbeiterinnen und Mitarbeiter sowie die Beiträgerinnen und Beiträger) mit entsprechend beharrlichem Druck vorangetrieben und in allen Phasen unterstützt haben. Vielmals danken möchte ich auch meinen Kölner und Gießener Studentinnen und Studenten, die mich mit ihrer intellektuellen Neugierde angespornt und immer wieder daran erinnert haben, wie groß der Bedarf an kompakten Überblicksdarstellungen über die Fülle von studienrelevanten literatur- und kulturtheoretischen Ansätzen und Grundbegriffen ist.

Meinen Gießener Mitarbeiterinnen und Mitarbeitern Sascha Feuchert, Nathalie Hahn, Sandra Heinen, Nora Lauck, Klaudia Seibel, Dagmar Sims, Roy Sommer und Bruno Zerweck sowie meiner Sekretärin Rosemary Lawson danke ich ganz herzlich für ihre enorme Einsatzbereitschaft, große Sorgfalt und die oftmals mühevolle Überprüfung sämtlicher Zitate und bibliographischer Angaben. Besonderer Dank gebührt Franziska Mosthaf und Carola Surkamp, die das Anliegen des Lexikons zu ihrer eigenen Sache gemacht und den Löwenanteil der Korrespondenz und redaktionellen Arbeiten mit der ihnen eigenen Akribie, Geduld, Gewissenhaftigkeit und Zuverlässigkeit erledigt haben. Das $\mathrm{Zu}$ standekommen dieses Lexikons ist nicht nur der engagierten Mitarbeit der Autorinnen und $\mathrm{Au}$ toren zu verdanken, sondern auch dem unermüdlichen Einsatz der namentlich Genannten und meiner Frau Vera.

Gießen, im April 1998

Ansgar Nünning 


\section{Vorwort zur zweiten Auflage}

$\mathrm{Da}$ dieses Lexikon schon drei Jahre nach dem Ersterscheinen in einer aktualisierten, überarbeiteten und erweiterten zweiten Auflage erscheint, ist der äußerst regen und positiven Aufnahme durch eine große Zahl von Leser/innen und Rezensent/innen zu verdanken. In der hier vorgelegten zweiten Auflage wurde die Konzeption der ersten beibehalten, aber es wurde eine Reihe von Ergänzungen und Aktualisierungen vorgenommen. Das Lexikon wurde um etwa 130 neue Artikel erweitert, die v.a. aus drei Bereichen stammen:

Erstens wurde die interdisziplinäre und intermediale Dimension der Theoriebildung sowohl durch zahlreiche neue Überblicksartikel als auch durch einige gezielte Nachträge noch stärker akzentuiert. Ergänzt wurden beispielsweise einige Theoretiker (u.a. Nelson Goodman, Emmanuel Lévinas, Humberto Maturana, Maurice Merleau-Ponty, Jean Piaget, Victor Turner) und etliche Grundbegriffe (z.B. Archiv, Dichte Beschreibung, Dispositiv, Emergenz, Identität, Interkulturalität, Liminalität, Performance/Performativität, Ritual, Theatralität, Transkulturation). Als übergreifende Artikel wurden neu aufgenommen: "Chaostheorie und Literatur", "Gedächtnis und Gedächtnistheorien", "Körpersprache in der Literatur", "Kulturanthropologie", "Modernisierung und Modernisierungstheorien", "Ecocriticism/Ökokritik", "Philosophie und Literatur", "Recht/Rechtssystem und Literatur", "Religion und Literatur", "Raum/ Raumdarstellung, literarische $(\mathbf{r})$ ", "Theater und Literatur", "Wirtschaft/Wirtschaftssystem und Literatur", "Zeit" und "Zivilisationstheorie".

Der damit unterstrichenen Weiterentwicklung der Philologien zu interdisziplinären Kultur- bzw. Medienkulturwissenschaften wurde zweitens durch einige Ergänzungen aus dem Bereich der Medientheorie Rechnung getragen (durch neue Artikel z.B. zu "Mediengattungstheorie" und "Medien und Literatur" sowie zu Medientheoretikern wie Béla Balázs, Vilém Flusser und Neil Postman und Theatertheoretikern wie Antonin Artaud und Martin Esslin). Aus Umfangsgründen sowie aufgrund der Tatsache, daß ein eigenes Metzler Lexikon Medientheorie und Medienwissenschaft in Vorbereitung ist, beschränken sich diese Erweiterungen jedoch auf einige gezielte Ergänzungen.
Drittens tragen eine Reihe von neu aufgenommenen Autorenporträts (z. B. Charles Baudelaire, Johann Gottfried Herder, Immanuel Kant, Gotthold Ephraim Lessing, Charles W. Morris) sowie Artikel zu den Literaturtheorien der Aufklärung und des Imagismus dazu bei, die historische Entwicklung der modernen Literatur- und Kulturtheorie, die bereits in der ersten Auflage durch viele literaturgeschichtliche Überblicksartikel zu den Literaturtheorien zentraler Epochen und Strömungen systematisch berücksichtigt wurde, noch umfassender und besser zu erschließen. Einem vielfach geäußerten Wunsch entsprechend, ist das Spektrum der Artikel durch neue Beiträge zur Schule der Annales und zu einigen auch für die Kulturtheorie wichtigen Geschichtstheoretikern (u.a. Philippe Ariès, Jacob Burckhardt, Roger Chartier, Natalie Zemon Davis und Jacques LeGoff) erweitert worden, um auch die Bezüge zwischen der Literatur- und Kulturtheorie und der Geschichtstheorie noch deutlicher zu konturieren.

Darüber hinaus wurden durch weitere Ergänzungen in allen Bereichen der Literatur- und Kulturtheorie gezielt Lücken geschlossen. Das Spektrum dieser Nachträge reicht von bislang fehlenden Grundbegriffen der Literaturtheorie (z.B. Chronotopos, Einbettung, Fiktionssignale, Foregrounding, Funktion, Groteske, Komik, Metadrama/Metatheater, Metalyrik, Montage/ Collage, Multiperspektivität, Offene vs. geschlossene Form, Schreibweisen, Spannung, Sprechsituation und Sympathielenkung) bis zu übergreifenden Konzepten aus benachbarten Disziplinen (z.B. Hermeneutik des Verdachts, Identitätstheorien, Rollentheorien, Stereotyp, Visualität und Visualitätskritik sowie die so aktuelle ,Weltentheories bzw. Possible-worlds theory).

Um dem gerade im Bereich der Theoriebildung besonders großen Bedürfnis nach aktueller Information $\mathrm{zu}$ entsprechen, sind außerdem sowohl die Auswahlbibliographie am Ende des Bandes als auch die bibliographischen Angaben einer Vielzahl von Artikeln aktualisiert worden. Die Veränderungen in den meisten Beiträgen der ersten Auflage beschränken sich ansonsten auf kleinere Korrekturen, die Bereinigung von formalen Unstimmigkeiten und einige sachliche Ergänzungen und Aktualisierungen. 
Die Einfügung neuer Verweislemmata (z.B. Bewußtseinsdarstellung $>$ Bewußtseinsstrom; $\nearrow$ Erlebte Rede; $\nearrow$ Fokalisierung; $\nearrow$ Gedankenbericht; $\gg$ Innerer Monolog) und weiterer Querverweise zwischen den Sachbegriffs- und Autorenartikeln sollen nicht nur die wissenschaftsgeschichtliche Einordnung erleichtern, sondern auch gewährleisten, daß Benutzer/innen gesuchte Aspekte gezielt nachschlagen und möglichst rasch auffinden können.

$*$

Den nunmehr etwa einhundertneunzig Autorinnen und Autoren, die an diesem Lexikon mitgearbeitet haben, sowie allen, die im Vorwort der ersten Auflage namentlich genannt sind, möchte ich nochmals ganz herzlich für die ausgezeichnete Zusammenarbeit und vielfältige Unterstützung danken. Für außerordentlich ermunternde Rückmeldungen und wertvolle Vorschläge danke ich einer Vielzahl von Kollegin- nen, Kollegen und Studierenden, deren Reaktionen mir in den letzten Jahren das Gefühl gegeben haben, daß sich all die Mühe gelohnt hat. Für wichtige Anregungen, die in der zweiten Auflage gerne berücksichtigt wurden, bin ich auch zahlreichen Rezensent/innen zu Dank verpflichtet, insbesondere Stefan Glomb, Günter Leypoldt, Andreas Mahler, Harald Neumeyer und vor allem Herbert Foltinek. Besonders großes Lob und Dank haben schließlich abermals meine Gießener Mitarbeiterinnen - Gaby Allrath, Wibke Bindemann, Britta Freitag, Stefanie Hoth, Rose Lawson, Nora Redhardt, Klaudia Seibel und vor allem Carola Surkamp - verdient, die mit ihrem unübertroffenen Engagement und großen Können unermüdlich halfen, einen möglichst fehlerfreien, druckreifen und optimalen Text zu erstellen, und die mich wieder in allen Phasen dieses Projekts unterstützt haben. Der größte Dank gebührt aber wie immer Vera.

Gießen, im Januar 2001

Ansgar Nünning 